\title{
Correlating Chest Surface Motion to Motion of the Liver Using $\varepsilon$-SVR - A Porcine Study
}

\author{
Floris Ernst ${ }^{1}$, Volker Martens ${ }^{1}$, Stefan Schlichting ${ }^{2}$, Armin Beširević ${ }^{2}$, \\ Markus Kleemann ${ }^{2}$, Christoph Koch ${ }^{3}$, Dirk Petersen ${ }^{3}$, and Achim Schweikard ${ }^{1}$ \\ ${ }^{1}$ Institute for Robotics and Cognitive Systems, University of Lübeck, DE \\ \{ernst, martens, schweikard\}@rob.uni-luebeck.de \\ ${ }^{2}$ Clinic for Surgery, University Hospital Schleswig-Holstein, Lübeck, DE \\ \{stefan.schlichting, armin. besirevic, markus.kleemann\}@uk-sh.de \\ ${ }^{3}$ Institute for Neuroradiology, University Hospital Schleswig-Holstein, Lübeck, DE \\ koch@neuroradiologie.uni-luebeck.de, dirk.petersen@uni-luebeck.de
}

\begin{abstract}
In robotic radiosurgery, the compensation of motion of internal organs is vital. This is currently done in two phases: an external surrogate signal (usually active optical markers placed on the patient's chest) is recorded and subsequently correlated to an internal motion signal obtained using stereoscopic X-ray imaging. This internal signal is sampled very infrequently to minimise the patient's exposure to radiation. We have investigated the correlation of the external signal to the motion of the liver in a porcine study using $\varepsilon$-support vector regression. IR LEDs were placed on the swines' chest. Gold fiducials were placed in the swines' livers and were recorded using a two-plane X-ray system. The results show that a very good correlation model can be built using $\varepsilon$ SVR, in this test clearly outperforming traditional polynomial models by at least 45 and as much as $74 \%$. Using multiple markers simultaneously can increase the new model's accuracy.
\end{abstract}

\section{Introduction}

In recent years, it has become possible to irradiate tumours in the whole body without using respiratory coaching, gating or stereotactic fixation. The CyberKnifeß [1] system - a robotic device used to detect and compensate for respiratory motion in radiosurgery - records optical markers placed on the patient's chest and correlates them to the position of landmarks, i.e. gold fiducials, obtained during stereoscopic X-ray imaging [2]. This model is subsequently used to guide a $\gamma$-radiation source. That this correlation indeed exists has been evaluated before 311. Currently, this model is built using 10-20 measurements of internal fiducials acquired during the first couple of breathing cycles. The model is checked and updated periodically. This is typically done once every 2-5 minutes by taking another X-ray shot. Furthermore, the markers (currently three) are placed on the patient's chest at those points showing the greatest excursion. The currently employed correlation model is either linear, curvilinear, dual-curvilinear or a mixture of those and is based on the principal directional component of motion of each individual chest LED [4].

G.-Z. Yang et al. (Eds.): MICCAI 2009, Part II, LNCS 5762, pp. 356-364, 2009.

(C) Springer-Verlag Berlin Heidelberg 2009 
We propose to improve the CyberKnife by modifying the correlation model employed in clinical practice. We compute the correlation using all three dimensions of movement of the LEDs as well as their first and second derivatives. This is done with a novel correlation model we have developed which is based on $\varepsilon$-support vector regression $(\varepsilon-\mathrm{SVR})$ [5]. With this model it is also possible to use multiple LEDs as input surrogates.

\section{Materials and Methods}

For this work, four gold fiducials were implanted into the liver of a living swine under US guidance. Respiratory motion of the liver was recorded in two sessions while the swine was ventilated manually using a bag valve mask. The swine was killed minutes prior to the experiments. An ethics proposal has been approved.

\subsection{Data Acquisition}

To acquire the fiducials' 3D position, a two-plane X-ray imaging device (Philips Allura Xper FD20/10, Fig. 1a at the Institute for Neuroradiology (University Hospital Schleswig-Holstein, Lübeck) was connected to a high resolution/high speed frame grabbing system (Matrox Helios XA) to allow the capturing of live fluoroscopic video streams. The X-ray system takes images at a frame rate of $15 \mathrm{~Hz}$. To record the swine's chest surface motion, a net of 19 IR LEDs (see 6] and Fig. 1b) was placed on the swine's abdomen. The LEDs were tracked using the atracsys accuTrack compact system, effectively delivering a recording frame rate of $216 \mathrm{~Hz}$ for each LED. The signals were then downsampled to $15 \mathrm{~Hz}$ to match the acquisition speed of the X-ray cameras.

To determine the geometric relation between the two X-ray imaging units and the tracking camera, a custom calibration rig was used: an acrylic box $(10 \times 7 \times 5$ $\mathrm{cm}^{3}$ ) with twelve embedded metallic spheres and eight LEDs was built. The system was calibrated by simultaneously acquiring an image of the calibration

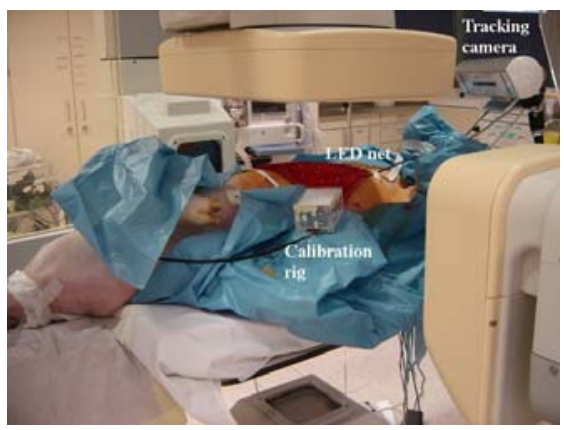

(a) Experimental setup

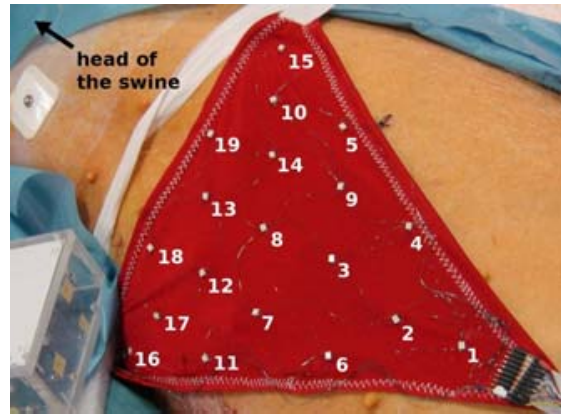

(b) The LED net

Fig. 1. X-ray device, tracking camera, calibration rig and LED net 

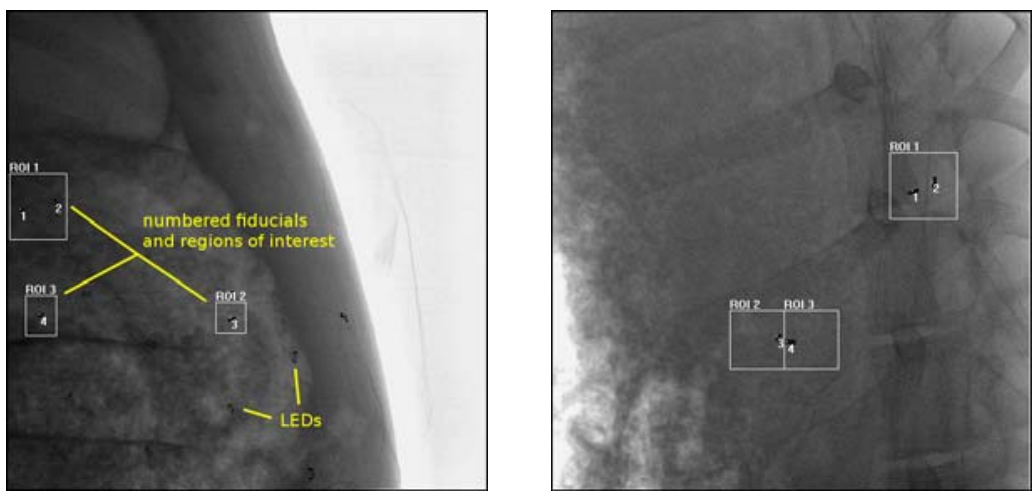

Fig. 2. The tracking GUI. Both the fiducials and the LEDs are clearly visible. The regions used for segmentation are marked with white rectangles.

rig with the X-ray devices and determining the rig's position using the tracking camera. The actual calibration was performed using the POSIT algorithm 7], resulting in a projection error of less than one pixel (RMS). Both the LEDs and the metal spheres could be detected with sub-millimetre accuracy.

The frame grabber and the IR tracking system are connected to one machine (Intel Q9450, 8 GiB RAM, ubuntu 8.04 x64). To track the gold fiducials in the $\mathrm{X}$-ray images, we developed a graphical tool kit written in $\mathrm{C}++$ to perform region-of-interest based segmentation of ellipsoidal objects and triangulate the 3D position of the fiducials (Fig. 2).

\subsection{Correlation Methods}

To compute the 3D position of the gold fiducials from the surrogate signal, the following methods were investigated:

- The polynomial models traditionally used in the CyberKnife, see [4]

- Our correlation model based on $\varepsilon$-SVR machines

These correlation algorithms were developed and implemented in MATLAB. The SVR machines were built using LibSVM [8].

Let us assume that $N$ is the number of samples we have taken. To build the correlation model, the input signal is divided into two parts: a training part $\mathcal{T}=\{1, \ldots, m\}$ and an evaluation part $\mathcal{E}=\{m+1, \ldots, N\}$. On the training part, we select points $\mathcal{M} \subseteq \mathcal{T}$ representative of the breathing pattern (i.e., points at maximum inspiration and expiration as well as points halfway between). Now let $\mathbf{L}_{i, j, n}$ be the time series of the 19 IR LEDs $(i=1, \ldots, 19$ is the number of the LED, $j=1, \ldots, 3$ are the spatial coordinates and $n$ is the temporal index) and let $\mathbf{F}_{k, j, n}$ be the time series of the four gold fiducials $(k=1, \ldots, 4$ is the fiducial number, $j$ and $n$ as before).

The polynomial models given in 4] are used to find coefficients of a linear or quadratic polynomial relating the principal directional component of motion of 
one LED to the principal directional component of motion of the fiducial. Additionally, the model supports breathing hysteresis by building two polynomials, one for inspiration and one for expiration. Both the simple quadratic model as well as the so-called bilinear and biquadratic models can also be blended to the simple linear model outside the values seen in the training data set $\mathcal{M}$.

We introduce a new correlation model based on $\varepsilon$-support vector regression. We do not only use the LEDs' principal directional component of motion as the polynomial models but all three dimensions. Second, information about the direction of breathing is directly built into the model by creating vectors $\mathbf{D}_{i}$ indicating the direction of breathing:

$$
\mathbf{D}_{i, n}=\left\{\begin{array}{cl}
-1 & \text { for } \tilde{\mathbf{L}}_{i, \cdot, n}-\tilde{\mathbf{L}}_{i, \cdot, n-1}<-0.05 m m \\
0 & \text { for }-0.05 m m \leq \tilde{\mathbf{L}}_{i, \cdot, n}-\tilde{\mathbf{L}}_{i, \cdot, n-1} \leq 0.05 m m, \quad n=2, \ldots, N \\
1 & \text { for } \tilde{\mathbf{L}}_{i, \cdot, n}-\tilde{\mathbf{L}}_{i, \cdot, n-1}>0.05 m m
\end{array}\right.
$$

Here, $\tilde{\mathbf{L}}_{i, \cdot, n}$ denotes the $n$-th sample of the principal directional component of the point cloud $\mathbf{L}_{i, .}$. Third, we incorporate information about the signal's speed and acceleration by also bringing in the first and second derivatives $\mathbf{L}^{(1)}$ and $\mathbf{L}^{(2)}$ of the LEDs' positions. These derivatives are computed using central differences. Now let $\mathbf{x}_{i, m}=\left(\mathbf{L}_{i, \cdot, m}^{\mathrm{T}}, \mathbf{L}_{i, \cdot, m}^{(1)}{ }^{\mathrm{T}}, \mathbf{L}_{i, \cdot, m}^{(2)}{ }^{\mathrm{T}}, \mathbf{D}_{i, m}\right)^{\mathrm{T}} \in \mathbb{R}^{9} \times\{-1,0,1\}$. Then for each $i=1, \ldots, 19, j=1, \ldots, 4$ and $m \in \mathcal{M}$ we create training samples $s_{m}^{i, j}=$ $\left\{\mathbf{x}_{i, m}, \tilde{\mathbf{F}}_{j, \cdot, m}\right\}$,i.e., the samples $s_{m}^{i, j}, m \in \mathcal{M}$, describe the relation between LED $i$ and $\tilde{F}_{j, .}$, the principal directional component of motion of fiducial $j$. These samples are then used to train $\varepsilon$-SVR machines which in turn serve as correlation models. The SVR machines are built using a linear kernel function.

\subsection{Evaluation}

The correlation models outlined above were applied to two groups of signals (120 $\mathrm{s}$ and $590 \mathrm{~s}$ duration) recorded during the ventilation of the swine. During both tests, not all LEDs were visible. In the first test, only LEDs 11, 14 and 16 to 19 were visible; in the second test, LEDs 6 to 14 and 16 to 19 were visible. The models were built using the first $20 \mathrm{~s}$ of motion. The $\varepsilon$-SVR's parameters were set to $C=1$ and $\varepsilon=0.2$ (signal 1$)$ and $\varepsilon=0.15$ (signal 2).

We also evaluated the influence of LED selection on correlation accuracy: first, which LED yields the best result and second, if the correlation model can be improved by using more than one LED at the same time.

\section{Results}

Analysis of LED motion shows that it is not only in one direction and does exhibit strong hysteresis relative to fiducial motion. This is the first indication that the simple polynomial models are not adequate. 


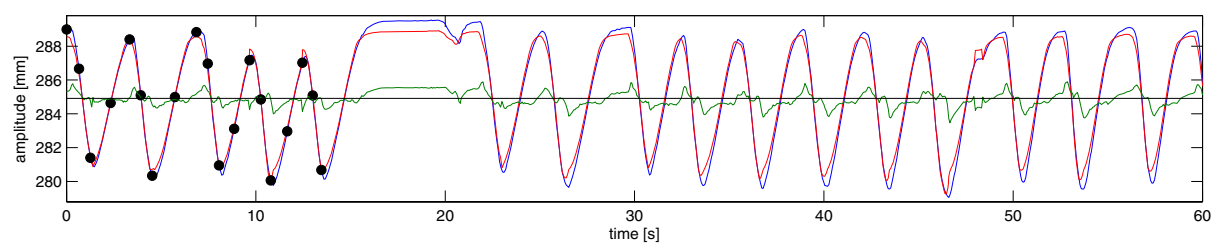

(a) The best polynomial model (biquadratic, blended)

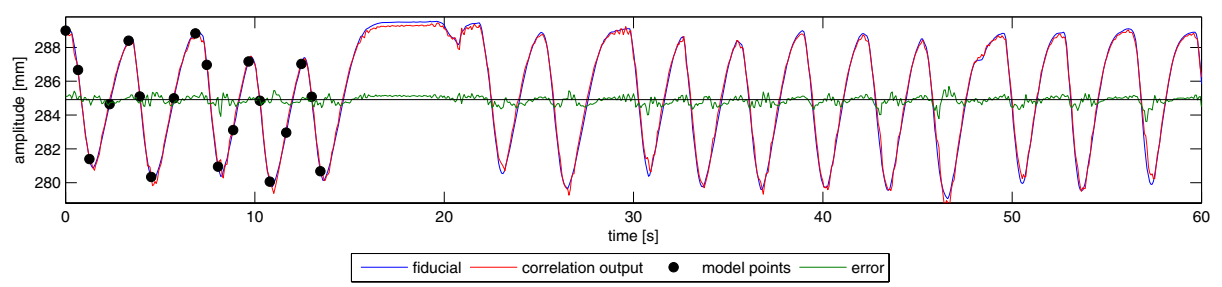

(b) Output of the $\varepsilon$-SVR model

Fig. 3. First test run, results of the correlation process. First $60 \mathrm{~s}$ are shown. Fiducial motion is shown in blue, training points used in black and the correlation output in red. The residual error is plotted in green. The respiratory pause around $t=20 \mathrm{~s}$ is accidental and not connected to the correlation model.

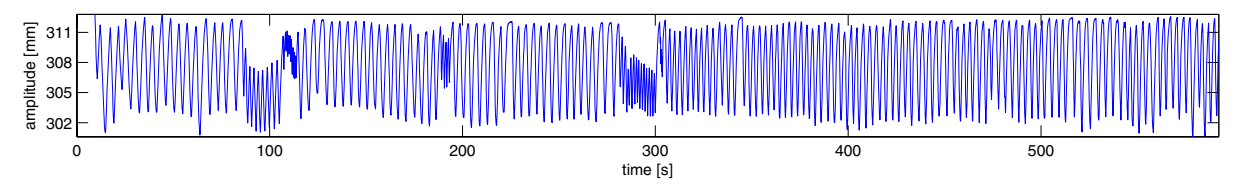

Fig. 4. Second test run. The signal shows variations in breathing frequency and amplitude.

The correlation plots of all the polynomial correlation models are given in Fig. 5. Ideally, the red curves would cover all blue dots. Clearly, the simple polynomial models don't fit the data very well, the bipolynomial models' matching is better. The actual numbers are given in Tab. 1

Evaluation results for the best polynomial model (biquadratic with blending) and for the $\varepsilon$-SVR model are given in Fig. 3. The polynomial model does not only incur a larger RMS error (see Tab. 1) but also suffers from periodic errors at the inspiration and expiration peaks. The reason for this is that the model does not adequately capture correlation in the regions marked with black dotted rectangles in Fig. $5 \mathrm{~g}$.

Evaluation of the $\varepsilon$-SVR correlation model shows a much better matching: Fig. 6] shows the correlation plots of the three axes of LED eleven versus the principal directional component of fiducial one. Clearly, the red dots (output of the correlation model) correspond very well to the blue dots (actual correlation). This is also reflected in the numbers given in Tab. 1 the SVR approach outperforms the best (bi)polynomial model by $45 \%$ (signal 1) or $38 \%$ (signal 
Table 1. RMS errors, $75 \%$ and $95 \%$ confidence intervals of the correlation models. Eleventh LED to first fiducial.

\begin{tabular}{l||c|c||c|c||c|c||c||c}
\multicolumn{1}{c||}{} & \multicolumn{3}{c||}{ RMS error [mm] } & \multicolumn{2}{c||}{$75 \%$ CI $[\mathrm{mm}]$} & $95 \%$ CI [mm] & \multicolumn{2}{c}{$\max [\mathrm{mm}]$} \\
& sig. 1 & sig. 2 & sig. 1 & sig. 2 & sig. 1 & sig. 2 & sig. 1 & sig. 2 \\
\hline \hline linear & 1.06 & 1.05 & 1.33 & 1.23 & 2.01 & 2.09 & 2.26 & 2.84 \\
quadratic & 1.03 & 1.04 & 1.32 & 1.22 & 1.90 & 2.04 & 2.14 & 2.80 \\
quadratic, blended & 1.03 & 1.04 & 1.32 & 1.22 & 1.90 & 2.04 & 2.14 & 2.80 \\
bilinear & 0.78 & 0.96 & 0.91 & 1.16 & 1.61 & 1.90 & 4.36 & 5.88 \\
bilinear, blended & 0.79 & 0.96 & 0.93 & 1.15 & 1.61 & 1.90 & 1.86 & 2.57 \\
biquadratic & 0.55 & 0.95 & 0.57 & 1.16 & 1.13 & 1.85 & 4.36 & 5.88 \\
biquadratic, blended & 0.50 & 0.95 & 0.52 & 1.14 & 1.05 & 1.85 & 1.71 & 2.54 \\
E-SVR & 0.28 & 0.59 & 0.28 & 0.64 & 0.52 & 1.12 & 1.48 & 4.14
\end{tabular}

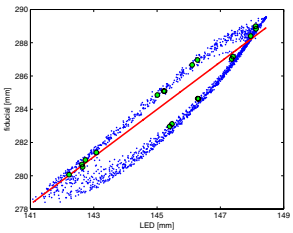

(a) linear

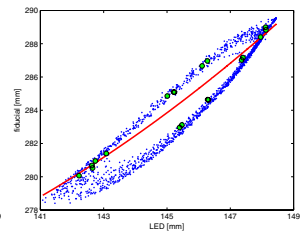

(b) quadratic

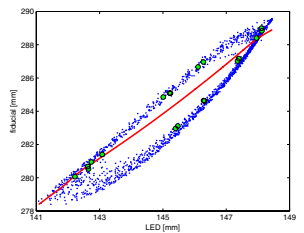

(c) quadr., blended

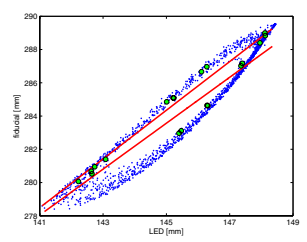

(d) bilinear

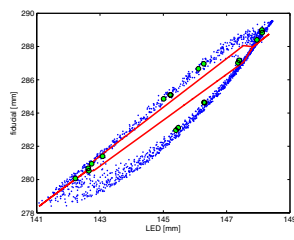

(e) bilinear, blended

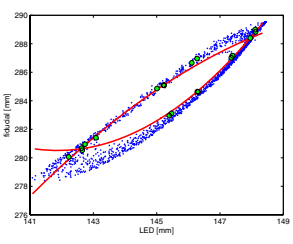

(f) biquadratic

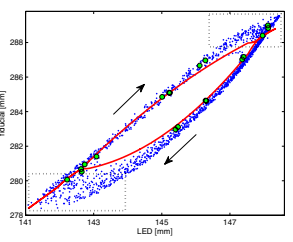

(g) biquad., blended

Fig. 5. The polynomial models. The x-axis shows the principal directional component of the motion of LED 11, the y-axis shows the principal directional component of motion of the first fiducial. Model points are marked with green circles, the model output is shown in red. In Subfig. (g), the dotted boxes show the areas corresponding to maximum inspiration and expiration; the arrows indicate the breathing direction.

2). Furthermore, the SVR model does not suffer from systematic errors like the polynomial models.

\subsection{Selection of LEDs and Using Multiple LEDs}

We also investigated the influence of LED selection on the quality of the correlation model. When selecting different LEDs as input surrogates, we see that on the first signal, the RMS error ranges from $0.27 \mathrm{~mm}$ to $0.38 \mathrm{~mm}$ whereas on the second signal, it ranges from $0.36 \mathrm{~mm}$ to $1.91 \mathrm{~mm}$. Since the $\varepsilon$-SVR correlation model has been designed such that it can use input from more than one LED at a time, we evaluated the model for all possible pairs (triplets, quadruplets, ...). The results are shown in Fig. 17. We can see that on the first signal, using more 

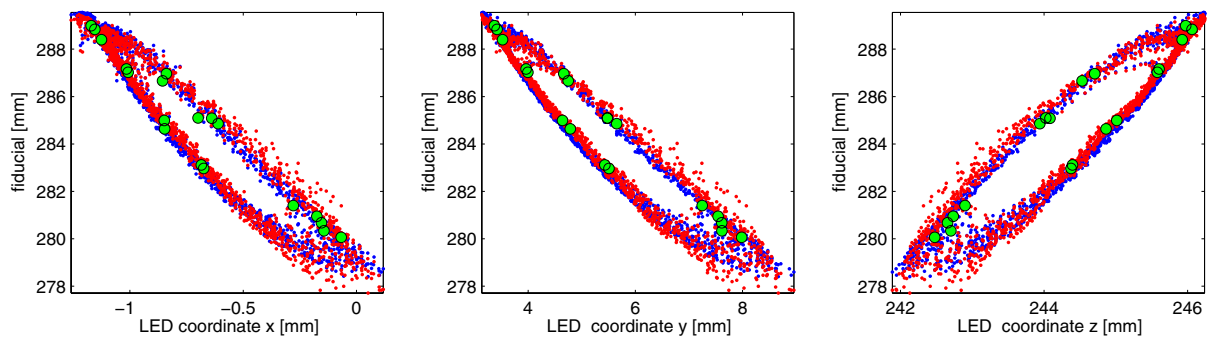

Fig. 6. The $\varepsilon$-SVR model. The $\mathrm{x}$-axes show the motion of LED 11, the y-axes show the principal directional component of motion of the first fiducial. Model points are marked with green circles, the model output is shown in red.

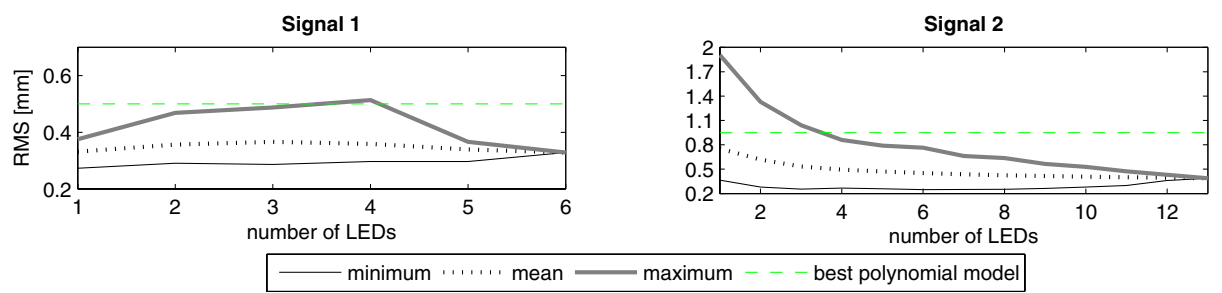

Fig. 7. The plots show the range (minimum and maximum values) and mean of the RMS error when using multiple LEDs. Read: when using six LEDs (1716 possibilites) to build the correlation model on signal two, the resulting RMS error is, depending on the selected sixtet, between 0.25 and $0.77 \mathrm{~mm}$ with a mean of $0.45 \mathrm{~mm}$.

than one LED does not noticeably improve correlation results, possibly due to overfitting. On the second signal, however, the best attainable correlation model uses six LEDs and outperforms the best model using one LED by $32 \%$ : the RMS value drops from $0.36 \mathrm{~mm}$ to $0.25 \mathrm{~mm}$. In this case, the best polynomial model is outperformed by as much as $74 \%$. Additionally, the $95 \%$ CI drops to $0.47 \mathrm{~mm}$. We believe that in this case we can clearly see that in this case the model using more LEDs is capable of catching the changing characteristics of the signal. Note that on signal one, only 19 and on signal two, only 17 samples are used to build the model which is evaluated on 1,876 and 8,588 samples, respectively.

\section{Discussion}

The fact that correlation on the second signal (with a length of $590 \mathrm{~s}$ ) can be improved significantly by using multiple LEDs is due to the signal's complexity: ventilation speed was varied as was the ventilation volume such as to mimick changing breathing patterns. It seems that especially the changes in breathing mode cannot be adequately covered by using one single LED.

We also went beyond the work presented in 910] and [1]. In [9], the internal position of the tumour was measured with NDI's magnetic tracking system 
using biopsy needles inserted into the swine. This approach suffers from poor temporal and spatial resolution and also from possibly altering the organ's motion patterns due to the insertion of biopsy needles. In 10, the evaluation of the correlation was done retrospectively on lung tumour patients where the tumour was located using stereoscopic fluoroscopy at changing angles and the external motion was recorded using a laser-based measuring device. The disadvantages in this study are the relatively short duration of the recordings (just over one minute in average) and the fact that the laser-based measuring system can only record distances and does not show the 3D displacement of a single point in space. In [11, the authors use very poor temporal resolution (4-5 Hz) and only compute the leave-one-out error of their correlation model. Furthermore, their model is built using all signal points but one and no long-term evaluation of the model is done.

In the near future, we plan to continue this study with more swines either under mechanical lung ventilation or breathing freely. We also hope to see this new correlation model implemented in the CyberKnife to perform tests under real conditions. Also, further investigation needs to be done as to where to place the LEDs on which the model is built, since this placement significantly influences the correlation accuracy.

\section{Conclusion}

It has been shown that the polynomial models used in determining the tumour position from chest marker positions are not very accurate, delivering RMS errors of up to $0.95 \mathrm{~mm}$, depending on the complexity of the input signal. On the other hand, the correlation model based on $\varepsilon$-SVR can achieve far better results: for both signals tested, RMS errors of $0.3 \mathrm{~mm}$ or less are feasible, a reduction of 40 to $75 \%$.

\section{References}

1. Adler, J.R., Schweikard, A., Murphy, M.J., Hancock, S.L.: Image-guided stereotactic radiosurgery: The CyberKnife. In: Image-guided neurosurgery: clinical applications of surgical navigation, pp. 193-204. Quality Medical Publishing (1998)

2. Schweikard, A., Glosser, G., Bodduluri, M., et al.: Robotic motion compensation for respiratory motion during radiosurgery. J. Comput. Aided Surg. 5(4), 263-277 (2000)

3. Gierga, D.P., Brewer, J., Sharp, G.C., et al.: The correlation between internal and external markers for abdominal tumors: Implications for respiratory gating. Int. J. Radiat. Oncol. Biol. Phys. 61(5), 1551-1558 (2005)

4. Sayeh, S., Wang, J., Main, W.T., et al.: Respiratory Motion Tracking for Robotic Radiosurgery. In: Urschel, H.C. (ed.) Robotic Radiosurgery. Treating Tumors that Move with Respiration, 1st edn., pp. 15-30. Springer, Berlin (2007)

5. Drucker, H., Burges, C.J.C., Kaufman, L., et al.: Support vector regression machines. In: Advances in Neural Information Processing Systems. NIPS, vol. 9, pp. 155-161. MIT Press, Cambridge (1997) 
6. Knöpke, M., Ernst, F.: Flexible Markergeometrien zur Erfassung von Atmungs und Herzbewegungen an der Körperoberfläche. In: 7. CURAC Jahrestagung, Leipzig, Germany, September 24-26, pp. 15-16 (2008)

7. DeMenthon, D.F., Davis, L.S.: Model-based object pose in 25 lines of code. Int. J. Comput. Vision 15(1-2), 123-141 (1995)

8. Chang, C.C., Lin, C.J.: LibSVM: a library for support vector machines (2001), http://www.csie.ntu.edu.tw/ cjlin/libsvm

9. Tang, J., Dieterich, S., Cleary, K.: Respiratory motion tracking of skin and liver in swine for CyberKnife motion compensation. In: Galloway Jr., R.L. (ed.) Medical Imaging 2004: Visualization, Image-Guided Procedures, and Display. vol. 5367, pp. 729-734. SPIE, San Diego (2004)

10. Seppenwoolde, Y., Berbeco, R.I., Nishioka, S., et al.: Accuracy of tumor motion compensation algorithm from a robotic respiratory tracking system: A simulation study. Med. Phys. 34(7), 2774-2784 (2007)

11. Khamene, A., Warzelhan, J.K., Vogt, S., Elgort, D., Chefd'Hotel, C., Duerk, J.L., Lewin, J.S., Wacker, F.K., Sauer, F.: Characterization of internal organ motion using skin marker positions. In: Barillot, C., Haynor, D.R., Hellier, P. (eds.) MICCAI 2004. Part II. LNCS, vol. 3217, pp. 526-533. Springer, Heidelberg (2004) 